\title{
Organizaciones de Significado Personal en adolescentes según el modelo posracionalista de Vittorio Guidano
}

\author{
Organizations of personal significance in adolescents according to the \\ postrationalist model of Vittorio Guidano
}

\author{
Marta Meza', Ismael Morales², Bárbara Cerda Aedo ${ }^{3}$
}

\begin{abstract}
RESUMEN
Objetivo: el trabajo analiza las Organizaciones de Significado Personal (OSP) según el modelo posracionalista de Guidano, en adolescentes. Metodología: enfoque cuantitativo, abarcando un estudio descriptivo, transversal, comparativo y correlacional. La muestra correspondió al total de alumnos de primer a cuarto año de enseñanza secundaria y se utilizó el instrumento de Evaluación de Relaciones de Significado Personal (OSP), que describe OSP: depresiva, dápica, fóbica y obsesiva. Resultados: Un 36,4\% de la muestra estudiada tiene una tendencia de OSP obsesiva. Siguiendo a esta, un 33,9\% tiene una tendencia de OSP fóbica. La prueba t demostró diferencias según género en las OSP depresiva $(p=0,007)$ y OSP dápica $(p=0,041)$. Conclusión: las OSP como constructo dinámico permiten analizar los procesos de apego dentro de la comunidad secundaria y dentro de los vínculos familiares.
\end{abstract}

Palabras clave: posracionalismo, apego, Organización de Significado Personal, adolescentes.

\begin{abstract}
Objective: the work analyzes the Organizations of Personal Meaning (OSP) according to the postrationalist model of Guidano, in adolescents. Methodology: quantitative approach, encompassing a descriptive, cross-sectional, comparative and correlational study. The sample corresponded to the total number of students from the first to the fourth year of secondary education and the instrument of Evaluation of Relationships of Personal Meaning (OSP) was used, which describes OSP: depressive, dápic, phobic and obsessive. Results: $36.4 \%$ of the studied sample has an obsessive OSP tendency. Following this, $33.9 \%$ have a phobic OSP trend. The t-test showed differences according to gender in depressive OSP $(p=0.007)$ and dpic OSP $(p=0.041)$. Conclusion: OSP as a dynamic construct allow the analysis of attachment processes within the secondary community and within family ties.
\end{abstract}

Keywords: post-rationalism, attachment, Organization of Personal Meaning, adolescents. 


\section{INTRODUCCIÓN}

La adolescencia es una etapa de transición, de transformaciones profundas en los sistemas comportamental, emocional y cognitivo (Allen \& Land, 1999). Los vínculos construidos con las figuras importantes de cuidado, durante los primeros años de vida, se constituyen como base para involucrarse en futuras relaciones. Por lo tanto, el desarrollo de un vínculo seguro en el niño es clave para su estado socioemocional, de lo contrario, según Bradley (como se citó en Agredano, 2018), una alteración socioemocional traducido en una conducta negativa y temerosa, con falta de empatía, desorganizada, con malas competencias sociales, baja autoestima, entre otros, podrían ser consideradas como factores de riesgo para desarrollar una patología infantil, y los niños con un vínculo inseguro estarían más expuestos a estas alteraciones. Entendiendo esto, un vínculo afectivo debe proporcionar al niño seguridad, intimidad y confianza como elementos claves (Ainsworth, 1991). Cuando se refiere específicamente a la adolescencia, dichos vínculos cobran importancia y se diversifican, de modo que es posible percibir cómo el apego desarrollado en la niñez se afianza y evoluciona hacia otros elementos vinculares relacionados con la confianza, puesto que más allá de las relaciones con los padres, para los adolescentes cobran relevancia y prioridad los vínculos con sus pares, entablando relaciones de carácter significativo con personas que no pertenecen al núcleo familiar (Carrillo, Castro, Penagos y Rodríguez, 2006), (Overbeek, Vollebergh, Engels y Meeus, 2003).

Respecto al fenómeno vincular, Guidano (1995) ahonda en la temática dentro del marco constructivista, elaborando el concepto de Organizaciones de Significado Personal (OSP), desarrolladas en etapas iniciales del ciclo vital a partir de los patrones de apego infantil y utilizando como referencia las clasificaciones tradicionales del vínculo afectivo (Arciero, 2000). Cuando Guidano se refiere a los cuatro tipos de OSP dápica, depresiva, fóbica y obsesiva, no se refiere a categorías ideales de alcanzar, sino a una manera de organizar la experiencia externa de acuerdo con la propia dimensión de coherencia que se ha elaborado. Con respecto a la OSP Depresiva, está caracterizada por una tendencia a responder con desesperanza ante eventos discordantes responsables de desequilibrio interno, en los cuales se elabora un significado para ello en torno a la pérdida; existiendo una naturaleza paradójica de la tristeza, que por un lado vincula a la persona con el mundo, y por otro lado le hace tomar distancia de este (Guidano, 1994). La OSP Fóbica, es la activación emocional reflejada en una sensación de temor a ser desprotegido y por ende una "castración" de la conducta exploratoria inicial, generando que el sujeto se muestre temeroso; manifestándose el deseo de ser protegido de un ambiente que se percibe como dañino y peligroso. (Guidano, 1987). La OSP Obsesiva, Sentido ambivalente de significados personales de todo o nada (Guidano, 1995); donde el individuo al encontrarse frente a necesidades de certezas absolutas crea una sensación de pérdida de control total, aflorando en él comportamientos e ideas de desequilibrio y caos que persisten más allá del deseo de la persona (Balbi, 1994). La OPS Dápica, Sentido difuso de sí mismo, que se organiza en el temor del cese de aprobación de otro o ser anulado por este, y al mismo tiempo en una constante búsqueda de dicha aprobación; un rasgo relevante de dichos sujetos es la modificación corporal disfuncional (trastornos alimenticios en general), fenómeno que se explica por la respuesta emocional que se desencadena cuando la persona interpreta un estímulo como una amenaza de rechazo inminente (Guidano, 1991) Considerando la importancia del vínculo afectivo en la niñez, la presente investigación pretende describir, correlacionar y comparar las Organizaciones de Significado Personal en adolescentes de 14 a 19 años según el modelo posracionalista.

Frente a esto, es que el objetivo principal es describir las Organizaciones de Significado Personal en adolescentes según el modelo posracionalista.

\section{METODOLOGÍA}

Enfoque cuantitativo, transversal, descriptivo, comparativo y correlacional.

La muestra no fue representativa de la población, por lo que correspondió al total de alumnos matriculados en el establecimiento municipal Colegio Comeweatlh School de Chillan, tomando como muestra a alumnos de primer a cuarto año medio de este establecimiento educacional, con edades que iban desde 14 a 19 años. Cada nivel posee un único curso, con excepción del segundo año medio que está 
formado por división A y B. Para efectos de la presente investigación, se consideró la totalidad de alumnos que comprendan los niveles ya mencionados.

Para la recolección de datos, el instrumento que se utilizó fue la Evaluación de Relaciones de Significado Personal de Vittorio Guidano, diseñado y validado por R. Cayupe y cols, el año 2013 en la Universidad de Chile. Este instrumento permite evaluar el tipo de organización de significado personal predominante en personas, a través de un cuestionario de autorreporte, que consta de 40 preguntas donde se analizan las OSP y como estas se manifiestan en la conformación de lazos afectivos y concepción del sí mismo en la adultez, donde se asignó el resultado de la OSP predominante sobre la base del mayor puntaje alcanzado en alguna de las variables.

Luego de aplicar el instrumento se analizaron los resultados con el software SPSSv.19, validando la consistencia interna con alfa de Cronbach, 0,858 para la OSP depresiva; 0,819 para la OSP dápica; 0,604 para OSP fóbica; 0,659 en OSP obsesiva.

En cuanto a los aspectos éticos de la investigación y en conformidad con la Ley $\mathrm{N}^{\circ}$ 19.628 de protección de la vida privada o de datos de carácter personal, los datos obtenidos en el cuestionario no han sido usados para ningún otro propósito fuera de los de esta investigación, siendo amparados por dicha ley.

Los sujetos de estudio fueron notificados acerca de la confidencialidad de la información obtenida mediante el instrumento de evaluación, así como tener también la oportunidad de hacer preguntas en relación con el estudio y recibir información de contacto en caso de desear realizar consultas en el futuro.

Los datos personales obtenidos en relación a la información personal fueron mantenidos en absoluta reserva, conservando el secreto profesional por parte del estudiante y docente guías del proceso investigativo. Una vez realizada la evaluación, esta información permaneció guarda en un lugar sin acceso a personas ajenas al estudio, y en ningún caso serán de conocimiento público. Los datos obtenidos no poseyeron identificación personal, por lo cual fueron usados y manipulados para la obtención de resultados en la investigación.
Finalmente, los participantes accedieron voluntariamente a participar de este estudio, sabiendo que podían retirarse mi consentimiento en cualquier momento de la investigación, sin penalización alguna.

\section{RESULTADOS}

La edad $(n=121)$ fue en promedio de 15,75 $+1,05$ años. Un $52,1 \%$ femenino, y un $47,9 \%$ masculino. Un $73,6 \%$ creció junto a su padre, y un $92,6 \%$ creció junto a su madre. Un $92,6 \%$ de la muestra vive actualmente con su madre, y un $72,7 \%$ con sus hermanos. Un $77,7 \%$ declara facilidad para hacer amigos. A la hora de conversar con desconocidos un 52,9\% afirma tener facilidad para entablar diálogo, teniendo en promedio 7,8 amigos cercanos. Un $36,4 \%$ de la muestra estudiada tiene una tendencia de OSP obsesiva. Siguiéndole un 33,9\% de OSP fóbica. Un 7,4\% tiene una tendencia de OSP dápica, y un $5,8 \%$ una tendencia de OSP depresiva. Con un 16,5 de tendencia mixta no definida.

La correlación de Pearson demostró relación entre los puntajes de las variables depresivo con dápico $(p<0,000 ; r=0,840)$, fóbico $(p<$ $0,000 ; r=0,549)$, obsesivo $(p=0,001 ; r=0,309)$. También hubo relación entre las variables dápico con fóbico $(p<0,000 ; r=0,597)$, obsesivo $(p<$ $0,000 ; r=0,379)$. Finalmente se relacionaron las variables obsesivo con fóbico $(p<0,000 ; r$ $=0,622$ )

Los estudiantes obtuvieron en la OSP depresiva una media de 20,02 puntos en mujeres y 17,02 en hombres, la prueba $t$ demostró diferencia entre ambos grupos $(p=0,007)$. En la OSP dápica se obtuvo una media de 20,55 puntos en mujeres y 18,45 en hombres, la prueba $t$ demostró diferencia entre ambos grupos $(p=$ 0,041 ). No hubo diferencias para los demás tipos de OSP. 


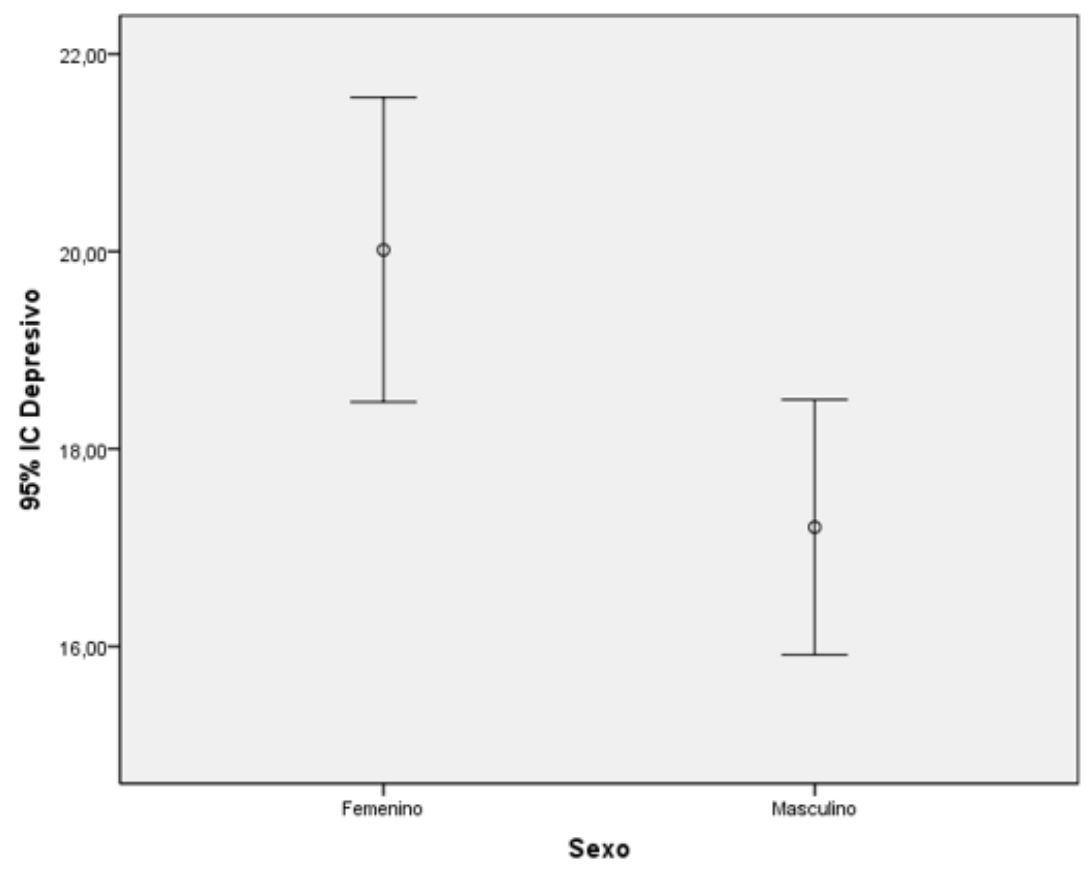

Figura 1

Estilo de OSP depresivo según sexo

La prueba $\mathrm{t}$ no demostró diferencias entre facilidad para hacer amigos y el puntaje obtenido en cada uno de los tipos de OSP $(p>0,05)$, como vivir actualmente con el padre $(p>0,05)$, ni diferencia estadística respecto a vivir con la madre $(p>0,05)$

Frente a la capacidad de conversar con desconocidos, un promedio de 18,14 puntos obtuvo los con "mayor facilidad" y 21,14 puntos quienes no. No presentándose diferencias significativas de promedio en los otros tipos de OSP $(p>0,05)$. (Figura 2)

\section{DISCUSIÓN}

Los adolescentes del colegio Comeweath School, con edades de entre 14 a 19 años presentaron una organización de significado personal predominante correspondiente a las OSP obsesiva $(36,4 \%)$ y fóbica $(33,9 \%)$, mientras que un menor porcentaje de ellos calificó en las categorías dápica $(7,4 \%)$ y depresiva (5,8\%). En cuanto a las correlaciones, los estudiantes con OSP depresivo tenían alta relación con la OSP dápica, moderada con la OSP fóbico y leve con OSP obsesivo, mientras que la relación entre obsesivo con fóbico fue media alta. Por lo tanto, podría encontrarse en un mismo perfil de adolescente de entre 14 a 19 años rasgos de desesperanza, desamparo, toma de distancia con el mundo y temor al cese de aprobación a la vez, como también en segunda instancia un perfil de adolescente donde el "todo o nada", la pérdida de control con el temor de ser desprotegido y la independencia podrían encontrarse en mayor medida dada su alta y media alta correlación.

La población adolescente estudiada tiene vínculos principalmente con su figura materna en el $92 \%$ de los casos, sin embargo, las pruebas $\mathrm{t}$ y r no demostraron diferencia ni relación entre el puntaje obtenido en las OSP y vivir actualmente con el padre o con la madre.

Se aprecia que un $36,4 \%$ de la muestra estudiada tiene tendencia de OSP obsesiva, siendo esta la organización predominante, es decir, en ella priman los perfiles de adolescentes donde la pérdida de control y falta de regulación de las emociones son comunes, lo cual sería normal para esa edad, sin embargo, se espera que esta conducta se vaya regulando con el tiempo, haciéndose cada vez más funcional y adaptativa. 
Respecto al contexto de desarrollo, Alarcón y Rosabal (1993), afirman que los adolescentes de colegios públicos tienden a establecer relaciones horizontales identificándose con su grupo de pares que con sus padres; muy a pesar del hecho que gran parte de la muestra creció junto a uno o ambos padres. Coté (2002), explica que jóvenes con una situación socioeconómico desfavorable se ven enfrentados a resolver solos mayor cantidad de tareas desde una edad más temprana, y por ende la identificación con las figuras de apego está dada desde el deber y la sensación de inseguridad que provoca el no saber si son o no amados, generando las bases de una OSP obsesiva.

\section{Correspondencia}

Ismael Morales Ojeda

Correo electrónico:

ismaelmorales@unach.cl

\section{REFERENCIAS}

Agredano, J. (2018). La familia como factor de riesgo para la psicopatología infantil.

Ainsworth, M. S. (1991). Attachments and other affectional bonds across the life cycle. En C. M. Parkes, J. Stevenson-Hinde y P. Marris (Eds.). Attachment across the life cycle (pp. 33-51). New York: Routledge.

Alarcón, M. y Rosabal, M. (1993). Conciencia moral e identidad del Yo. Estudio de casos en dos grupos de adolescentes de diferentes clases sociales. Una propuesta teórico metodológica. Tesis de licenciatura en Psicología, Universidad de Costa Rica, Costa Rica.

Allen, J. \& Land, D. (1999). Attachment in adolescence. En J. Cassidy y P.

Allen, J. P. (2008). The attachment system in adolescence. En J. Cassidy y P. R. Shaver (Eds.), Handbook of attachment: Theory, research and clinical applications (pp. 419-435). New York: The Guildford Press.

Allen, J. P.; McElhaney, K. B.; Kuperminc, G. P. y Jodl, K. M. (2004). Stability and change in attachment security across adolescence. Child Development, 75(6), 1792-1805. Recuperado de: https://www. ncbi.nlm.nih.gov/pmc/articles/PMC1761126/pdf/ nihms11560.pdf
Arciero, G. (2000). Las organizaciones de personalidad: El enfoque post racionalista. Revista de Psicoterapia, 11(41), 93-102.

Balbi, J. (1994). Terapia cognitiva post racionalista: Conversaciones con Vittorio Guidano. Buenos Aires, Argentina: Editorial Biblos.

Carrillo, S.; Castro, J.; Penagos, A. y Rodriguez, M. (2006). Apego, relaciones románticas y auto concepto en adolescentes bogotanos. Psychol, 5(1), 21-36.

Cook, W. L. (2000). Understanding attachment security in family context. Journal of Personality and Social Psychology, 78, 285-294.

Coté, J. (2002). The role of identity capital in the transition to adulthood: The individualization thesis examined. Journal of Youth Studies, 5(2), 117-134.

Garreaud, A. M. y González, M. (1998). "Tranquilidad artificial". La teoría de Vittorio Guidano sobre las organizaciones de significado personal y su aplicación en un grupo de mujeres consumidoras de Benzodiazepinas, sin prescripción de especialistas COSAM Maipú, Chile 1997. Tesis de licenciatura en Psicología, Universidad Académica de Humanismo Cristiano, Santiago, Chile.

Guidano, V. (1987). La complejidad de uno mismo: Un enfoque evolutivo de la psicopatología y la psicoterapia. New York: Guilford Press.

Guidano, V. (1991). El sí mismo en proceso. Barcelona, España: Editorial Paidós.

Guidano, V. (1994). La terapia cognitiva desde una perspectiva evolutivo constructivista. Roma, Italia: Centro de Terapia Cognitiva de Roma.

Guidano, V. (1995). Desarrollo de la terapia cognitiva post racionalista. Santiago, Chile: Editorial Instituto de Terapia Cognitiva.

Hayek, F. (1952). The sensory order. Chicago: University of Chicago Press.

Hazan, C. y Zaifman, D. (2008). Pair bonds as attachments: Reevaluating the evidence. En J. Cassidy y P. R. Shaver (Eds.), Handbook of attachment: Theory, research and clinical applications (pp. 436-455). New York: The Guildford Press.

Lundy, B.; Field, T.; Lundy, B.; McBride, C. y Largie, S. (1998). Same-sex and opposite-sex best friend interactions among high school juniors and seniors. Adolescence, 33(130), 279-289. 
Moltedo, A. y Onetto, L. (2002). Las Organizaciones de Significado Personal de Vittorio Guidano: Una llave explicativa de la experiencia humana. Psicoperspectivas, 1(1), 83-92. Recuperado de: http://www.cognitivimarca.altervista.org/ Download/oneto_moltedo.pdf

Nardi, B. (2006). Rol de los procesos filogenéticos y ontogenéticos en el desarrollo de las organizaciones de significado personal. Psicoperspectivas, 5(1). (49-64).

Overbeek, G.; Vollebergh, W.; Engels, C. y Meeus, W. (2003). Parental attachment and romantic relationships: Associations with emotional disturbance during late adolescence. Journal of Counseling Psychology, 50(1), 28-39.
Shaver (Eds) Handbook of attachment. Theory, research and clinical applications, 319-335. Nueva York: The Guilford Press.

Streitmatter, J. (1989). Ethnicity as a mediating variable of early adolescent identity development. Journal of Adolescence, 11(4), 335-346.

Thompson, R. A. (2000). The legacy of early attachments. Child Development, 71(1), 145152. Recuperado de: http://people.uncw.edu/ hungerforda/Graduate\%20Developmental/PDF/ thompson 2000.pdf

Recibido: 30/06/2021

Aceptado: 23/09/2021 